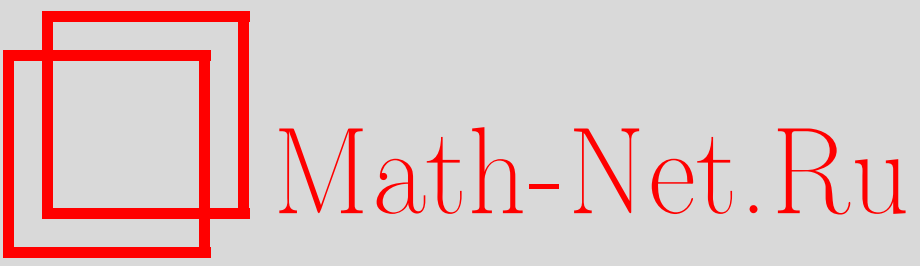

В. Ю. Матвеев, Бесконечная алгебраическая независимость некоторых почти полиадических чисел, Итоги науки и техн. Сер. Соврем. мат. и ее прил. Темат. обз., 2020, том 179, 29-33

DOI: https://doi.org/10.36535/0233-6723-2020-179-29-33

Использование Общероссийского математического портала Math-Net.Ru подразумевает, что вы прочитали и согласны с пользовательским соглашением

http://www.mathnet.ru/rus/agreement

Параметры загрузки:

IP: 52.6 .47 .48

26 апреля 2023 г., 07:34:13 


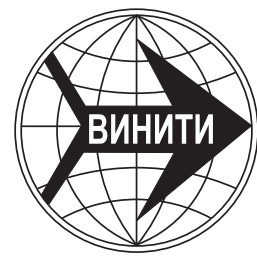

ИТОГИ НАУКИ И ТЕХНИКИ.

Современная математика и ее приложения.

Тематические обзоры.

Том 179 (2020). С. 29-33

DOI: 10.36535/0233-6723-2020-179-29-33

УДК 511.22

\title{
БЕСКОНЕЧНАЯ АЛГЕБРАИЧЕСКАЯ НЕЗАВИСИМОСТЬ НЕКОТОРЫХ ПОЧТИ ПОЛИАДИЧЕСКИХ ЧИСЕЛ
}

\author{
(C) 2020 г. B. Ю. MATBEEB
}

АннотАция. В статье рассматриваются вопросы бесконечной алгебраической независимости полиадических и почти полиадических чисел.

Ключевые слова: полиадические числа, почти полиадические числа, бесконечная алгебраическая независимость.

\section{INFINITE ALGEBRAIC INDEPENDENCE OF SOME ALMOST POLYADIC NUMBERS}

\author{
(c) 2020 V. YU. MATVEEV
}

\begin{abstract}
In this paper, we discuss some problems of infinite algebraic independence of polyadic and almost polyadic numbers.

Keywords and phrases: polyadic number, almost polyadic number, infinite algebraic independence.

AMS Subject Classification: 11J91,11J61
\end{abstract}

1. Полиадические и почти полиадические числа. Рассмотрим множество точек вида $\left(x_{1}, x_{2}, \ldots, x_{n}, \ldots\right)$, образующих бесконечномерное пространство. Это пространство представляет собой прямое произведение полей радических чисел. Именно, координата с номером $n$ этого вектора представляет собой $p_{n}$ адическое число, где $p_{n}$ - простое число с номером $n$. Напомним, что радические числа являются пополнением поля рациональных чисел по радическому нормированию. Для рационального числа $a$ символ $|a|_{p}$ обозначает величину радического нормирования, т.е. $|a|_{p}=p^{-\vartheta_{p}(a)}$, где, в свою очередь, символ $\vartheta_{p}(a)$ обозначает степень, в которой простое число $p$ входит в разложение числа $a$ на простые множители. Это прямое произведение имеет естественную структуру коммутативного кольца с единицей (и с делителями нуля). Его принято называть кольцом полиадических чисел. Элементы a кольца целых полиадических чисел имеют каноническое представление в виде

$$
\mathfrak{a}=\sum_{m=1}^{\infty} a_{m} m !, \quad a_{m} \in \mathbb{N}, \quad 0 \leqslant a_{m} \leqslant m .
$$

Степень, в которой простое число $p$ входит в разложение числа $n$ ! на простые множители, равна $\frac{n-S_{n}}{p-1}$, где $S_{n}$ обозначает сумму цифр в $p$-ичном разложении числа $n$. Следовательно, для любого $p$ при $n \rightarrow \infty$ выполняется соотношение $\left|a_{n} n !\right|_{p} \rightarrow 0$. Это - достаточное условие для того, чтобы ряд (1) сходился в поле $\mathbb{Q}_{p}$. Сумму этого ряда $(1)$ в поле $\mathbb{Q}_{p}$ обозначаем $\mathfrak{a}^{(p)}$. Как отмечено выше, полиадическое число $\mathfrak{a}$ можно рассматривать как точку бесконечномерного пространства с координатами $\mathfrak{a}^{\left(p_{n}\right)}$, где $p_{n}-$ простое число с номером $n$. 
Следует отметить, что ряды вида $\sum_{n=0}^{\infty} a_{n} n !, a_{n} \in \mathbb{Q}$ могут сходиться не во всех полях $\mathbb{Q}_{p}$. В случае, когда такой ряд расходится лишь в конечном множестве полей $\mathbb{Q}_{p}$, мы говорим о почти полиадических числах и отождествляем их с элементами прямого произведения всех полей $\mathbb{Q}_{p}$, кроме упомянутого выше конечного множества. Примером служит ряд

$$
\sum_{n=0}^{\infty}(\Lambda, n), \quad \Lambda=\frac{a}{b}, a \in \mathbb{Z}, b \in \mathbb{N},
$$

где величина $(\Lambda, n)$ определена равенствами

$$
(\Lambda, 0)=1, \quad(\Lambda, n)=(\Lambda)(\Lambda+1) \ldots(\Lambda+n-1), \quad n \geqslant 1 .
$$

Действительно, по теореме Дирихле о простых числах в арифметической прогрессии, для любого простого числа $p$, не делящего числа $b$,

$$
|\Lambda, n|_{p}=\left|\frac{(a)(a+b) \ldots(a+b(n-1))}{b^{n}}\right|_{p} \rightarrow 0, \quad n \rightarrow \infty .
$$

Можно рассмотреть и прямое произведение полей $\mathbb{K}_{v}$, где $v$ продолжает $p$-адическое нормирование поля $\mathbb{Q}$ на поле $\mathbb{K}$ алгебраических чисел конечной степени $\varkappa$. Если $v$ продолжает $p$-адическое нормирование, то поле $\mathbb{K}_{v}$ представляет собой алгебраическое расширение поля $p$-адических чисел $\mathbb{Q}_{p}$ степени $\varkappa_{v}$, и справедливо равенство $\sum_{v} \varkappa_{v}=\varkappa$, где суммирование производится по всем нормированиям $v$, продолжающим $p$-адическое нормирование. Мы рассматриваем нормализованные нормирования, для которых $|p|_{v}=p^{-\frac{\varkappa v}{\varkappa}}$. Если нормирование $v$ продолжает обычную абсолютную величину, то ему соответствует алгебраически сопряженное поле $\mathbb{K}^{(i)}$, и $\varkappa_{v}=1$, если $\mathbb{K}^{(i)}$ - подполе поля действительных чисел, и $\varkappa_{v}=2$, если нет. Равенство $\sum_{v} \varkappa_{v}=\varkappa$ выполняется
и в этом случае.

При этом мы будем говорить о $\mathbb{K}$-полиадических и, соответственно, о $\mathbb{K}$-nочти полиадических числах. Для элемента $\mathfrak{a}$ этого прямого произведения обозначаем $\mathfrak{a}^{(v)}$ его координату в поле $\mathbb{K}_{v}$.

В работах В. Г. Чирского $[11,15,16]$ предложена следующая классификация арифметических свойств вышеупомянутых классов чисел.

Если существует такой многочлен $P(x)$ с рациональными коэффициентами, отличный от тождественного нуля, что $P(\mathfrak{a})=0$ (иными словами, $P\left(\mathfrak{a}^{(v)}\right)=0$ в каждом поле $\mathbb{K}_{v}$ этого прямого произведения), то говорим, что $\mathfrak{a}$-алгебраический элемент. Если элемент $\mathfrak{a}$ не является алгебраическим, то его называют трансцендентным. Трансцендентность элемента означает, что для любого $P(x)$ - многочлена с рациональными коэффициентами, отличного от тождественного нуля, существует простое число $p$ и нормирование $v$ поля $\mathbb{K}$, продолжающее $p$-адическое нормирование поля $\mathbb{Q}$ такие, что $P\left(\mathfrak{a}^{(v)}\right) \neq 0$ в поле $\mathbb{K}_{v}$. Назовем элемент a бесконечно трансцендентным, если для любого $P(x)$ - многочлена с рациональными коэффициентами, отличного от тождественного нуля, существует бесконечное множество простых чисел $p$, для каждого из которых есть нормирование $v$ поля $\mathbb{K}$, продолжающее $p$-адическое нормирование, такое, что $P\left(\mathfrak{a}^{(v)}\right) \neq 0$ в поле $\mathbb{K}_{v}$. Элемент $\mathfrak{a}$ называется глобально трансцендентным, если для любого $P(x)$ - многочлена с рациональными коэффициентами, отличного от тождественного нуля, неравенство $P\left(\mathfrak{a}^{(v)}\right) \neq 0$ выполняется во всех полях $\mathbb{K}_{v}$ рассматриваемого прямого произведения.

2. Бесконечная алгебраическая независимость некоторых рядов. Для исследования арифметических свойств значений степенных рядов применяется метод Зигеля-Шидловского в теории трансцендентных чисел (см. $[19,21])$. Этот метод применим к широким классам рядов, так называемым $E$ - и $G$-функциям Зигеля. Обобщение метода применимо к $F$-рядам.

Пусть

$$
f(z)=\sum_{n=0}^{\infty} c_{n} \cdot n ! \cdot z^{n} .
$$

Будем говорить, что этот ряд принадлежит классу $F\left(\mathbb{K}, C_{1}, C_{2}, C_{3}, q\right)$, если: 
1. Все коэффициенты $c_{n}$ принадлежат некоторому алгебраическому числовому полю $\mathbb{K}$ конечной степени $\varkappa$ над полем $\mathbb{Q}$ рациональных чисел.

2. Максимумы абсолютных величин алгебраически сопряженных с числом $c_{n}$ чисел представляют собой $O\left(e^{C_{1} n}\right), n \rightarrow \infty$ с некоторой постоянной $C_{1}$.

3. Существует последовательность натуральных чисел $d_{n}$ такая, что при $k=0,1, \ldots, n$ числа $d_{n} c_{k}$ принадлежат кольцу целых чисел $\mathbb{Z}_{\mathbb{K}}$ поля $\mathbb{K}$ и $d_{n}=q^{n} d_{0, n}, q \in \mathbb{N}$, а числа $d_{0, n}$ делятся только на простые числа $p$, не превосходящие $C_{2} n$, и для всех таких простых $p$ выполняется неравенство $\vartheta_{p}\left(d_{0, n}\right) \leqslant C_{3}\left(\log _{p} n+n / p^{2}\right)$.

Арифметические свойства $F$-рядов исследованы в $[1,6-12,15,16,20]$. Сформулируем используемую далее теорему.

Теорема 1. Пусть F-ряды $f_{1}(z), f_{2}(z), \ldots, f_{m}(z)$ составляют решение системъ

$$
y_{i}^{\prime}=Q_{i, 0}(z)+\sum_{j=1}^{m} Q_{i, j}(z) y_{j}, \quad i=1, \ldots, m
$$

с коэффициентами из поля $\mathbb{C}(z)$ рачиональных функиий от $z$ и алгебраически независимы над полем $\mathbb{K}(z)$. Пусть $\xi \in \mathbb{K}$ - регулярная точка системъ (3). Пусть $P\left(y_{1}, \ldots, y_{m}\right)$ - ненулевой многочлен с коэффициентами из $\mathbb{Z}_{\mathbb{K}}$. Тогда существует бесконечное множество таких простых чисел р и нормирований $v$ поля $\mathbb{K}$, продолжающих р-адическое нормирование поля $\mathbb{Q}$, что в поле $\mathbb{K}_{v}$

$$
P\left(f_{1}(\xi), \ldots, f_{m}(\xi)\right) \neq 0 .
$$

Иными словами, ряды $f_{1}(\xi), \ldots, f_{m}(\xi)$ бесконечно алгебраически независимы.

Теорема 2. Пусть $f_{1}(z), \ldots, f_{m}(z)$ представляют собой трансцендентные $F$-ряды с иельми коэфбиииентами, составляющие решение системы вида

$$
P_{1, i} y_{i}^{\prime}+P_{0, i} y_{i}=Q_{i} ; \quad i=1, \ldots, m ; \quad P_{0, i}, P_{1, i}, Q_{i} \in \mathbb{Q}(z) .
$$

Пусть $\xi \in \mathbb{K}, \xi$ отлично от 0 и особых точек системы, а также выполняются следующие условия:

$$
\exp \left(\int\left(\frac{P_{0, i}(z)}{P_{1, i}(z)}-\frac{P_{0, j}(z)}{P_{1, j}(z)}\right) d z\right) \notin \mathbb{C}(z), \quad i \neq j .
$$

Тогда почти полиадические числа $\alpha_{1}=f_{1}(\xi), \ldots, f_{m}(\xi)=\alpha_{m}$ бесконечно алгебраччески независимы.

Доказательство теоремы 2 сводится к применению сформулированной выше теоремы и следующей теоремы.

Теорема 3. Пусть $f_{1}(z), \ldots, f_{m}(z)$ представляют собой трансиендентные F-ряды с иелыми коэффициентами, составляющие решение системы вида

$$
P_{1, i} y_{i}^{\prime}+P_{0, i} y_{i}=Q_{i}, \quad i=1, \ldots, m ; \quad P_{0, i}, P_{1, i}, Q_{i} \in \mathbb{Q}(z) .
$$

Пусть выполняются следующие условия:

$$
\exp \left(\int\left(\frac{P_{0, i}(z)}{P_{1, i}(z)}-\frac{P_{0, j}(z)}{P_{1, j}(z)}\right) d z\right) \notin \mathbb{C}(z), \quad i \neq j .
$$

Тогда рлды $f_{1}(z), \ldots, f_{m}(z)$ алгебраччески независимы над $\mathbb{C}(z)$.

Доказательство теоремы 3 получено методом В. Х. Салихова (см. [4]). Приложением теоремы 3 является следующее утверждение.

Теорема 4. Почти полиадические числа $\mathfrak{a}_{i}$, определенные равенствами

$$
\sum_{n=1}^{\infty} a_{i} \xi^{n}\left(a_{i}+b_{i}\right) \ldots\left(a_{i}+(n-1) b_{i}\right)=\mathfrak{a}_{i},
$$


где $a_{i}, b_{i} \in \mathbb{Z},\left(a_{i}, b_{i}\right)=1, i=1, \ldots, m$,

$$
\frac{a_{i}}{b_{i}}-\frac{a_{j}}{b_{j}} \notin \mathbb{Z}, \quad i \neq j
$$

бесконечно алгебраически независимы.

Используя аппроксимации Эрмита-Паде, можно доказать следующую теорему.

Теорема 5. Пусть $\xi-$ полиадическое число, обладающее следующими свойствами:

(i) существует бесконечное множество таких полиадических чисел $\tau_{s}, s \in \mathbb{N}$, что

$$
\xi=\beta_{s}+\tau_{s}, \quad \beta_{s} \in \mathbb{N}, \quad \beta_{s} \leqslant C_{1} e^{\sqrt{\ln s}}
$$

(ii) для всех простых чисел $p, p \leqslant a+b s$, выполнено неравенство

$$
\left|\tau_{s}\right|_{p}=\left|\xi-\beta_{s}\right|_{p}<e^{-s \ln s-2 s \sqrt{\ln s}-C_{2} s} .
$$

Тогда существует бесконечное множсество таких простых чисел р, что в поле $\mathbb{Q}_{p}$ справедливо неравенство

$$
1+\sum_{n=1}^{\infty} \lambda(\lambda+1) \ldots(\lambda+(n-1)) \xi^{n} \neq 0 .
$$

Эта теорема является следствием теоремы 4, но позволяет получить несколько более точные оценки для интервалов, в которых содержатся упоминаемые в теореме простые числа $р$. Упомянутые выше теоремы справедливы и для полиадических точек $\xi$, допускающих высокие порядки приближения натуральными числами.

3. Некоторые применения полиадических и почти полиадических чисел. Полиадические числа используются в ряде задач аналитической теории чисел (см. [3]). Они также используются в ряде задач, связанных с теорией абелевых групп (см. [5]). Частичные суммы рядов (1) представляют собой так называемые факториальные разложения натуральных чисел. Для этих разложений доказана оценка количества получающихся слагаемых лучшая, чем известная оценка для двухбазисных разложений. Однако практические вычисления показывают, что обычно двухбазисные разложения несколько короче факториального разложения. Однако факториальные разложения короче разложения в двухбазисную цепь (см. [17]).

Наконец, цифры конечных сумм рядов вида (1) обладают хорошими статистическими свойствами в смысле псевдослучайности (см. [18]).

\section{СПИСОК ЛИТЕРАТУРЫ}

1. Матвеев В. Ю., Чирский В. Г. О некоторых свойствах полиадических разложений// Чебышев. сб. 2013. - 14, № 2. - C. 164-172.

2. Матвеев В. Ю. Алгебраическая независимость некоторых почти полиадических рядов// Чебышев. сб. - 2016. - 17, № 3. - С. 166-177.

3. Постников А. Г. Введение в аналитическую теорию чисел. - М.: Наука, 1971.

4. Салихов B. Х. Об алгебраической независимости значений $E$-функций, удовлетворяющих линейным дифференциальным уравнениям первого порядка// Мат. заметки. - 1973. - 13, № 1. - С. 29-40.

5. Фомин A. А. Числовые кольца и модули над ними. - Москва: Прометей, 2013.

6. Чирский В. Г. О рядах, алгебраически независимых во всех локальных полях// Вестн. Моск. ун-та. Cер. 1. Мат. Мех. - 1978. - № 3. - C. 29-34.

7. Чирский В. Г. О глобальных соотношениях// Мат. заметки. - 1990. - 48, № 2. - С. 123-127.

8. Чирский В. Г. Об алгебраических соотношениях в неархимедовски нормированных полях// Функц. анал. прилож. - 1992. - 26, № 2. - С. 41-50.

9. Чирский В. Г. Метод Зигеля в р-адической области// Фундам. прикл. мат. - 2005. - 11, № 6. C. 221-230.

10. Чирский В. Г. Обобщение понятия глобального соотношения// Зап. науч. семин. ПОМИ. - 2005. 322. - C. 220-238. 
11. Чирский В. Г. Арифметические свойства полиадических рядов с периодическими коэффициентами// Докл. РАН. - 2014. - 459, № 6. - С. 677-679.

12. Чирский В. Г. Об арифметических свойствах обобщенных гипергеометрических рядов с иррациональными параметрами// Изв. РАН. Сер. мат. - 2014. - 78, № 6. - С. 193-210.

13. Чирский В. Г. Арифметические свойства целых полиадических чисел// Чебышев. сб. - 2015. - 16, № 1. - C. 254-264.

14. Чирский В. Г. Об арифметических свойствах ряда Эйлера// Вестн. Моск. ун-та. Сер. 1. Мат. Мех. 2015. - № 1. - C. 59-61.

15. Чирский В. Г. Арифметические свойства полиадических рядов с периодическими коэффициентами// Изв. РАН. Сер. мат. - 2017. - 81, № 2. - С. 215-232.

16. Чирский В. Г. Арифметические свойства обобщенных гипергеометрических $F$-рядов// Докл. РАН. 2018. - 483, № 3. - C. 252-254.

17. Чирский В. Г., Матвеев В. Ю. О представлении натуральных чисел// Чебышев. сб. - 2013. - 14, № 1. - C. $75-86$.

18. Чирский В. Г., Матвеев В. Ю. О некоторых свойствах полиадических разложений// Чебышев. сб. 2013. - 14, № 2 (46). - C. 164-172.

19. Шидловский А. Б. Трансцендентные числа. - М.: Наука, 1987.

20. Bertrand D., Chiskii V., Yebbou J. Effective estimates for global relations on Euler-type series// Ann. Fac. Sci. Toulouse. - 2004. - 13, № 2. - P. 241-260.

21. Chirskii V. G. Topical problems of the theory of transcendental numbers: Developments of approaches to tyeir solutions in works of Yu. V. Nesterenko// Russ. J. Math. Phys. — 2017. — 24, № 2. - P. 153-171.

Матвеев Владимир Юрьевич

Московский педагогический государственный университет

E-mail: salomaa@mail.ru 